\title{
Strong convergence of a CQ method for $k$-strictly asymptotically pseudocontractive mappings
}

\author{
Hossein Dehghan ${ }^{1}$ and Naseer Shahzad ${ }^{2 *}$
}

\section{"Correspondence:}

nshahzad@kau.edu.sa

${ }^{2}$ Department of Mathematics, King

Abdulaziz University, P.O. Box 80203,

Jeddah, 21589, Saudi Arabia

Full list of author information is

available at the end of the article

\begin{abstract}
Let $E$ be a real $q$-uniformly smooth Banach space, which is also uniformly convex (for example, $L_{p}$ or $\ell_{p}$ spaces, $\left.1<p<\infty\right)$, and $C$ be a nonempty bounded closed convex subset of $E$. Let $T: C \rightarrow C$ be a k-strictly asymptotically pseudocontractive map with a nonempty fixed point set. A hybrid algorithm is constructed to approximate fixed points of such maps. Furthermore, strong convergence of the proposed algorithm is established.
\end{abstract}

Keywords: strong convergence; CQ method; $k$-strictly asymptotically pseudocontractive mapping

\section{Introduction}

Let $E$ be a real Banach space and $E^{\prime \prime}$ be the dual of $E$. We denote the value of $x^{\prime \prime} \in E^{\prime \prime}$ at $x \in E$ by $\left\langle x, x^{*}\right\rangle$. The normalized duality mapping $J$ from $E$ to $2^{E^{*}}$ is defined by

$$
J(x)=\left\{x^{*} \in E^{*}:\left\langle x, x^{*}\right\rangle=\|x\|^{2}=\left\|x^{*}\right\|^{2}\right\}
$$

for all $x \in E$. It is known that a Banach space $E$ is smooth if and only if the normalized duality mapping $J$ is single valued. Some properties of the duality mapping have been given in $[1,2]$.

Let $C$ be a nonempty subset of $E$. The mapping $T: C \rightarrow C$ is called nonexpansive if

$$
\|T x-T y\| \leq\|x-y\|
$$

for all $x, y \in C$. Also, $T$ is called uniformly L-Lipschitz if there exists a constant $L>0$ such that

$$
\left\|T^{n} x-T^{n} y\right\| \leq L\|x-y\|
$$

for all $x, y \in C$ and each $n \geq 1$. The mapping $T: C \rightarrow C$ is called $k$-strictly asymptotically pseudocontractive if there exist a sequence $\left\{k_{n}\right\}$ in $[1, \infty)$ with $\lim _{n \rightarrow \infty} k_{n}=1$ and a constant $k \in[0,1)$, and for any $x, y \in C$, there exists $j(x-y) \in J(x-y)$ such that

$$
\left\langle T^{n} x-T^{n} y, j(x-y)\right\rangle \leq \frac{1}{2}\left(1+k_{n}\right)\|x-y\|^{2}-\frac{1}{2}(1-k)\left\|x-T^{n} x-\left(y-T^{n} y\right)\right\|^{2}
$$


for each $n \geq 1$. If $I$ denotes the identity operator, then (1.1) can be written in the form

$$
\begin{aligned}
\left\langle\left(I-T^{n}\right) x-\left(I-T^{n}\right) y, j(x-y)\right) \geq & \frac{1}{2}(1-k)\left\|\left(I-T^{n}\right) x-\left(I-T^{n}\right) y\right\|^{2} \\
& -\frac{1}{2}\left(k_{n}-1\right)\|x-y\|^{2} .
\end{aligned}
$$

The class of $k$-strictly asymptotically pseudocontractive mappings was first introduced in Hilbert spaces by Qihou [3]. In Hilbert spaces, $j$ is the identity and it is shown [4] that (1.1) (and hence (1.2)) is equivalent to the inequality

$$
\left\|T^{n} x-T^{n} y\right\|^{2} \leq k_{n}\|x-y\|^{2}+k\left\|\left(I-T^{n}\right) x-\left(I-T^{n}\right) y\right\|^{2}
$$

which is the inequality considered by Qihou [3]. In the same paper, the author proved strong convergence of the modified Mann iteration processes for $k$-strictly asymptotically pseudocontractive mappings in Hilbert spaces. The modified Mann iteration scheme was introduced by Schu [5, 6] and has been used by several authors (see, for example, [7-12]). In [13] Osilike extended Qihou's result from Hilbert spaces to much more general real $q$-uniformly smooth Banach spaces, $1<q<\infty$.

The classes of nonexpansive and asymptotically nonexpansive mappings are important classes of mappings because they have applications to solutions of differential equations which have been studied by several authors (see, e.g., [14-16] and references contained therein). It would be of interest to study the class of $k$-strictly asymptotically pseudocontractive mappings in view of the fact that it is closely related to the above two classes.

On the other hand, using the metric projection, Matsushita and Takahashi [17] introduced the following iterative algorithm for nonexpansive mappings: $x_{0}=x \in C$ and

$$
\left\{\begin{array}{l}
C_{n}=\overline{c 0}\left\{z \in C:\|z-T z\| \leq t_{n}\left\|x_{n}-T x_{n}\right\|\right\} \\
D_{n}=\left\{z \in C:\left\langle x_{n}-z, J\left(x-x_{n}\right)\right\rangle \geq 0\right\} \\
x_{n+1}=P_{C_{n} \cap D_{n}} x, \quad n=0,1,2, \ldots
\end{array}\right.
$$

where $\overline{c o} D$ denotes the convex closure of the set $D, J$ is the normalized duality mapping, $\left\{t_{n}\right\}$ is a sequence in $(0,1)$ with $t_{n} \rightarrow 0$, and $P_{C_{n} \cap D_{n}}$ is the metric projection from $E$ onto $C_{n} \cap D_{n}$. Then, they proved that $\left\{x_{n}\right\}$ generated by (1.3) converges strongly to a fixed point of the mapping $T$.

In this paper, motivated by these facts, we introduce the following iterative algorithm for finding fixed points of a $k$-strictly asymptotically pseudocontractive mapping $T$ in a uniformly convex and $q$-uniformly smooth Banach space: $x_{1}=x \in C, C_{0}=D_{0}=C$ and

$$
\left\{\begin{array}{l}
C_{n}=\overline{\operatorname{co}}\left\{z \in C_{n-1}:\left\|z-T^{n} z\right\| \leq t_{n}\left\|x_{n}-T^{n} x_{n}\right\|\right\} \\
D_{n}=\left\{z \in D_{n-1}:\left\langle x_{n}-z, J\left(x-x_{n}\right)\right\rangle \geq 0\right\} \\
x_{n+1}=P_{C_{n} \cap D_{n}} x, \quad n=1,2, \ldots
\end{array}\right.
$$

where $\overline{\mathrm{co}} D$ denotes the convex closure of the set $D, J$ is the normalized duality mapping, $\left\{t_{n}\right\}$ is a sequence in $(0,1)$ with $t_{n} \rightarrow 0$, and $P_{C_{n} \cap D_{n}}$ is the metric projection from $E$ onto $C_{n} \cap D_{n}$. 
The purpose of this paper is to establish a strong convergence theorem of the iterative algorithm (1.4) for $k$-strictly asymptotically pseudocontractive mappings in a uniformly convex and $q$-uniformly smooth Banach space.

\section{Preliminaries}

The modulus of smoothness of a Banach space $E$ is the function $\rho_{E}:[0, \infty) \rightarrow[0, \infty)$ defined by

$$
\rho_{E}(t)=\sup \left\{\frac{1}{2}(\|x+y\|+\|x-y\|)-1:\|x\| \leq 1,\|y\| \leq t\right\} .
$$

$E$ is uniformly smooth if and only if $\lim _{t \rightarrow 0^{+}} \rho_{E}(t) / t=0$. Let $q>1$. The Banach space $E$ is said to be q-uniformly smooth if there exists a constant $c>0$ such that $\rho_{E}(t) \leq c t^{q}$. Hilbert spaces, $L_{p}$ (or $\ell_{p}$ ) spaces, $1<p<\infty$, and the Sobolev spaces, $W_{m}^{p}, 1<p<\infty$, are $q$-uniformly smooth.

When $\left\{x_{n}\right\}$ is a sequence in $E$, we denote strong convergence of $\left\{x_{n}\right\}$ to $x \in E$ by $x_{n} \rightarrow x$ and weak convergence by $x_{n} \rightarrow x$. The Banach space $E$ is said to have the Kadec-Klee property if for every sequence $\left\{x_{n}\right\}$ in $E, x_{n} \rightarrow x$ and $\left\|x_{n}\right\| \rightarrow\|x\|$ imply that $x_{n} \rightarrow x$. Every uniformly convex Banach space has the Kadec-Klee property [1].

Let $C$ be a nonempty closed convex subset of a reflexive, strictly convex, and smooth Banach space $E$. Then for any $x \in E$, there exists a unique point $x_{0} \in C$ such that

$$
\left\|x_{0}-x\right\|=\min _{y \in C}\|y-x\|
$$

The mapping $P_{C}: E \rightarrow C$ defined by $P_{C} x=x_{0}$ is called the metric projection from $E$ onto $C$. Let $x \in E$ and $u \in C$. Then it is known that $u=P_{C} x$ if and only if

$$
\langle u-y, J(x-u)\rangle \geq 0
$$

for all $y \in C$ ( see $[1,18])$.

In the sequel, we need the following results.

Proposition 2.1 (See [19]) Let C be a bounded closed convex subset of a uniformly convex Banach space E. Then there exists a strictly increasing convex continuous function $\gamma:[0, \infty) \rightarrow[0, \infty)$ with $\gamma(0)=0$ depending only on the diameter of $C$ such that

$$
\gamma\left(\left\|\sum_{i=1}^{n} \lambda_{i} T x_{i}-T\left(\sum_{i=1}^{n} \lambda_{i} x_{i}\right)\right\|\right) \leq \max _{1 \leq i<j \leq n}\left(\left\|x_{i}-x_{j}\right\|-\left\|T x_{i}-T x_{j}\right\|\right)
$$

holds for any nonexpansive mapping $T: C \rightarrow E$, any elements $x_{1}, \ldots, x_{n}$ in $C$, and any numbers $\lambda_{1}, \ldots, \lambda_{n} \geq 0$ with $\lambda_{1}+\cdots+\lambda_{n}=1$.

Corollary 2.2 [20, Corollary 1.2] Under the same suppositions as in Proposition 2.1, there exists a strictly increasing convex continuous function $\gamma:[0, \infty) \rightarrow[0, \infty)$ with $\gamma(0)=0$ depending only on the diameter of $C$ such that

$$
\gamma\left(\left\|\sum_{i=1}^{n} \lambda_{i} x_{i}-T\left(\sum_{i=1}^{n} \lambda_{i} x_{i}\right)\right\|\right) \leq \max _{1 \leq i \leq n}\left(\left\|x_{i}-T x_{i}\right\|\right)
$$


holds for any nonexpansive mapping $T: C \rightarrow E$, any elements $x_{1}, \ldots, x_{n}$ in $C$, and any numbers $\lambda_{1}, \ldots, \lambda_{n} \geq 0$ with $\lambda_{1}+\cdots+\lambda_{n}=1$. (Note that $\gamma$ does not depend on $T$.)

In order to utilize Corollary 2.2 for $k$-strictly asymptotically pseudocontractive mappings, we need the following lemmas.

Lemma 2.3 [4] Let $E$ be a real Banach space, $C$ be a nonempty subset of $E$, and $T$ : $C \rightarrow C$ be a $k$-strictly asymptotically pseudocontractive mapping. Then $T$ is uniformly $L$ Lipschitzian.

Lemma 2.4 [21, Lemma 3.1] Let $E$ be a real q-uniformly smooth Banach space and $C$ be a nonempty convex subset of $E$. Let $T: C \rightarrow C$ be a $k$-strictly asymptotically pseudocontractive map, and let $\left\{\alpha_{n}\right\}$ be a real sequence in $[0,1]$. Define $S_{n}: C \rightarrow C$ by $S_{n} x:=$ $\left(1-\alpha_{n}\right) x+\alpha_{n} T^{n} x$ for all $x \in C$. Then for all $x, y \in C$, we have

$$
\begin{aligned}
\left\|S_{n} x-S_{n} y\right\|^{q} \leq & \left(1+\frac{q}{2} \alpha_{n}\left(k_{n}-1\right)\right)\|x-y\|^{q} \\
& -\alpha_{n}\left(\frac{q}{2}(1-k)(1+L)^{-(q-2)}-c_{q} \alpha_{n}^{q-1}\right)\left\|\left(I-T^{n}\right) x-\left(I-T^{n}\right) y\right\|^{q},
\end{aligned}
$$

where $L$ is the uniformly Lipschitzian constant of $T$ and $c_{q}>0$ is the constant which appeared in [21, Theorem 2.1].

Let $\beta=\min \left\{1,\left[\frac{q}{2}(1-k)(1+L)^{-(q-2)} / c_{q}\right]^{1 /(q-1)}\right\}$ and choose $\alpha \in(0, \beta)$. Set $\alpha_{n}=\alpha$ for all $n \geq 1$ in Lemma 2.4 and observe that $\left\|S_{n} x-S_{n} y\right\|^{q} \leq\left(1+\frac{q}{2} \alpha\left(k_{n}-1\right)\right)\|x-y\|^{q}$. Thus,

$$
\left\|S_{n} x-S_{n} y\right\| \leq\left(1+\frac{q}{2} \alpha\left(k_{n}-1\right)\right)^{1 / q}\|x-y\|
$$

for all $x, y \in C$ and each $n \geq 1$.

Theorem 2.5 [21, Theorem 3.1] Let E be a real q-uniformly smooth Banach space which is also uniformly convex. Let $C$ be a nonempty closed convex subset of $E$ and $T: C \rightarrow C$ be a $k$-strictly asymptotically pseudocontractive mapping with a nonempty fixed point set. Then $(I-T)$ is demiclosed at zero, i.e., if $x_{n} \rightarrow x$ and $x_{n}-T x_{n} \rightarrow 0$, then $x \in F(T)$, where $F(T)$ is the set of all fixed points of $T$.

\section{Strong convergence theorem}

In this section, we study the iterative algorithm (1.4) for finding fixed points of $k$-strictly asymptotically pseudocontractive mappings in a uniformly convex and $q$-uniformly smooth Banach space. We first prove that the sequence $\left\{x_{n}\right\}$ generated by (1.4) is well defined. Then, we prove that $\left\{x_{n}\right\}$ converges strongly to $P_{F(T)} x$, where $P_{F(T)}$ is the metric projection from $E$ onto $F(T)$.

Lemma 3.1 Let $C$ be a nonempty closed convex subset of a reflexive, strictly convex, and smooth Banach space $E$, and let $T: C \rightarrow C$ be a mapping. If $F(T) \neq \emptyset$, then the sequence $\left\{x_{n}\right\}$ generated by $(1.4)$ is well defined. 
Proof It is easy to check that $C_{n} \cap D_{n}$ is closed and convex and $F(T) \subset C_{n}$ for each $n \in \mathbb{N}$. Moreover, $D_{1}=C$ and so $F(T) \subset C_{1} \cap D_{1}$. Suppose $F(T) \subset C_{k} \cap D_{k}$ for $k \in \mathbb{N}$. Then there exists a unique element $x_{k+1} \in C_{k} \cap D_{k}$ such that $x_{k+1}=P_{C_{k} \cap D_{k}} x$. If $u \in F(T)$, then it follows from (2.1) that

$$
\left\langle x_{k+1}-u, J\left(x-x_{k+1}\right)\right\rangle \geq 0,
$$

which implies $u \in D_{k+1}$. Therefore, $F(T) \subset C_{k+1} \cap D_{k+1}$. By the mathematical induction, we obtain that $F(T) \subset C_{n} \cap D_{n}$ for all $n \in \mathbb{N}$. Therefore, $\left\{x_{n}\right\}$ is well defined.

In order to prove our main result, the following lemma is needed.

Lemma 3.2 Let $C$ be a nonempty bounded closed convex subset of a real q-uniformly smooth and uniformly convex Banach space E. Let $T: C \rightarrow C$ be a $k$-strictly asymptotically pseudocontractive mapping with $\left\{k_{n}\right\}$ such that $F(T) \neq \emptyset$. Let $\left\{x_{n}\right\}$ be the sequence generated by (1.4), then for any $j \in \mathbb{N}$,

$$
\lim _{n \rightarrow \infty}\left\|x_{n}-T^{n-j} x_{n}\right\|=0
$$

Proof Fix $j \in \mathbb{N}$ and put $m=n-j$. Since $x_{n}=P_{C_{n-1} \cap D_{n-1}} x$, we have $x_{n} \in C_{n-1} \subseteq \cdots \subseteq C_{m}$. Since $t_{m}>0$, there exist $y_{1}, \ldots, y_{N} \in C$ and $\lambda_{1}, \ldots, \lambda_{N} \geq 0$ with $\lambda_{1}+\cdots+\lambda_{N}=1$ such that

$$
\left\|x_{n}-\sum_{i=1}^{N} \lambda_{i} y_{i}\right\|<t_{m}
$$

and $\left\|y_{i}-T^{m} y_{i}\right\| \leq t_{m}\left\|x_{m}-T^{m} x_{m}\right\|$ for all $i \in\{1, \ldots, N\}$. It follows from Lemma 2.3 that $T$ is uniformly $L$-Lipschitzian. Put $M=\sup _{x \in C}\|x\|, u=P_{F(T)} x$ and $r_{0}=\sup _{n \geq 1}(1+L)\left\|x_{n}-u\right\|$. Thus,

$$
\left\|y_{i}-T^{m} y_{i}\right\| \leq t_{m}\left\|x_{m}-T^{m} x_{m}\right\| \leq t_{m}(1+L)\left\|x_{m}-u\right\| \leq r_{0} t_{m}
$$

for all $i \in\{1, \ldots, N\}$. Define $H_{m}: C \rightarrow E$ by

$$
H_{m} x=\frac{1}{a_{m}} S_{m} x
$$

for all $x \in C$, where $a_{m}=\left(1+\frac{q}{2} \alpha\left(k_{m}-1\right)\right)^{1 / q}$ and $S_{m}$ is as in (2.2). It follows from (2.2) that $H_{m}$ is nonexpansive. Using (3.2) and the fact that $\left\|y_{i}-S_{m} y_{i}\right\|=\alpha\left\|y_{i}-T^{m} y_{i}\right\|$, we have

$$
\left\|y_{i}-H_{m} y_{i}\right\| \leq\left(1-\frac{1}{a_{m}}\right)\left\|y_{i}\right\|+\frac{1}{a_{m}}\left\|y_{i}-S_{m} y_{i}\right\| \leq\left(1-\frac{1}{a_{m}}\right) M+\alpha r_{0} t_{m}
$$

for all $i \in\{1, \ldots, N\}$. It follows from Corollary 2.2, (3.1), and (3.3) that

$$
\begin{aligned}
\left\|x_{n}-H_{m} x_{n}\right\| \leq & \left\|x_{n}-\sum_{i=1}^{N} \lambda_{i} y_{i}\right\|+\left\|\sum_{i=1}^{N} \lambda_{i} y_{i}-H_{m}\left(\sum_{i=1}^{N} \lambda_{i} y_{i}\right)\right\| \\
& +\left\|H_{m}\left(\sum_{i=1}^{N} \lambda_{i} y_{i}\right)-H_{m} x_{n}\right\|
\end{aligned}
$$




$$
\begin{aligned}
& \leq 2 t_{m}+\gamma^{-1}\left(\max _{1 \leq i \leq N}\left\|y_{i}-H_{m} y_{i}\right\|\right) \\
& \leq 2 t_{m}+\gamma^{-1}\left(\left(1-\frac{1}{a_{m}}\right) M+\alpha r_{0} t_{m}\right)
\end{aligned}
$$

Since $\lim _{n \rightarrow \infty} a_{n}=1$ and $\lim _{n \rightarrow \infty} t_{n}=0$, it follows from the last inequality that $\lim _{n \rightarrow \infty} \| x_{n}-$ $H_{m} x_{n} \|=0$. Thus, $\lim _{n \rightarrow \infty}\left\|x_{n}-S_{m} x_{n}\right\|=0$ and so $\lim _{n \rightarrow \infty}\left\|x_{n}-T^{m} x_{n}\right\|=0$. This completes the proof.

Theorem 3.3 Let $C$ be a nonempty bounded closed convex subset of a real q-uniformly smooth and uniformly convex Banach space E. Let $T: C \rightarrow C$ be a k-strictly asymptotically pseudocontractive mapping with $\left\{k_{n}\right\}$ such that $F(T) \neq \emptyset$. Let $\left\{x_{n}\right\}$ be the sequence generated by (1.4). Then $\left\{x_{n}\right\}$ converges strongly to the element $P_{F(T)}$ of $F(T)$, where $P_{F(T)}$ is the metric projection from $E$ onto $F(T)$.

Proof Put $u=P_{F(T)} x$. Since $F(T) \subset C_{n} \cap D_{n}$ and $x_{n+1}=P_{C_{n} \cap D_{n}} x$, we have that

$$
\left\|x-x_{n+1}\right\| \leq\|x-u\|
$$

for all $n \in \mathbb{N}$. By Lemma 3.2, we have

$$
\begin{aligned}
\left\|x_{n}-T x_{n}\right\| & \leq\left\|x_{n}-T^{n-1} x_{n}\right\|+\left\|T^{n-1} x_{n}-T x_{n}\right\| \\
& \leq\left\|x_{n}-T^{n-1} x_{n}\right\|+L\left\|T^{n-2} x_{n}-x_{n}\right\| \rightarrow 0 \quad \text { as } n \rightarrow \infty
\end{aligned}
$$

Since $\left\{x_{n}\right\}$ is bounded, there exists $\left\{x_{n_{i}}\right\} \subset\left\{x_{n}\right\}$ such that $x_{n_{i}} \rightarrow v$. It follows from Theorem 2.5 (demiclosedness of $T$ ) that $v \in F(T)$. From the weakly lower semicontinuity of norm and (3.4), we obtain

$$
\|x-u\| \leq\|x-v\| \leq \liminf _{i \rightarrow \infty}\left\|x-x_{n_{i}}\right\| \leq \limsup _{i \rightarrow \infty}\left\|x-x_{n_{i}}\right\| \leq\|x-u\|
$$

This together with the uniqueness of $P_{F(T)} x$ implies $u=v$, and hence $x_{n_{i}} \rightarrow u$. Therefore, we obtain $x_{n} \rightarrow u$. Furthermore, we have that

$$
\lim _{n \rightarrow \infty}\left\|x-x_{n}\right\|=\|x-u\|
$$

Since $E$ is uniformly convex, using the Kadec-Klee property, we have that $x-x_{n} \rightarrow x-u$. It follows that $x_{n} \rightarrow u$. This completes the proof.

The authors declare that they have no competing interests.

\section{Authors' contributions}

All authors contributed equally and significantly in writing this article. All authors read and approved the final manuscript.

\section{Author details}

'Department of Mathematics, Institute for Advanced Studies in Basic Sciences (IASBS), Gava Zang, Zanjan, 45137-66731, Iran. ${ }^{2}$ Department of Mathematics, King Abdulaziz University, P.O. Box 80203, Jeddah, 21589, Saudi Arabia. 


\section{Acknowledgements}

The authors are grateful to the reviewers for their useful comments.

\section{Received: 6 February 2012 Accepted: 26 October 2012 Published: 22 November 2012}

\section{References}

1. Agarwal, RP, Regan, DO, Sahu, DR: Convexity, smoothness and duality mappings. In: Fixed Point Theory for Lipschitzian-type Mappings with Applications, pp. 49-115. Springer, New York (2009)

2. Cioranescu, I: Geometry of Banach Spaces, Duality Mappings and Nonlinear Problems. Kluwer Academic, Dordrecht (1990)

3. Qihou, L: Convergence theorems of the sequence of iterates for asymptotically demicontractive and hemicontractive mappings. Nonlinear Anal. 26(11), 1835-1842 (1996)

4. Osilike, MO, Aniagbosor, SC, Akuchu, BG: Fixed points of asymptotically demicontractive mappings in arbitrary Banach spaces. Panam. Math. J. 12(2), 77-88 (2002)

5. Schu, J: Iterative construction of fixed point of asymptotically nonexpansive mappings. J. Math. Anal. Appl. 158 407-413 (1991)

6. Schu, J: Weak and strong convergence to fixed points of asymptotically nonexpansive mappings. Bull. Aust. Math Soc. 43, 153-159 (1991)

7. Ofoedu, EU: Strong convergence theorem for uniformly L-Lipschitzian asymptotically pseudocontractive mapping in real Banach space. J. Math. Anal. Appl. 321(2), 722-728 (2006)

8. Rafiq, A: On iterations for families of asymptotically pseudocontractive mappings. Appl. Math. Lett. 24, 33-38 (2011)

9. Osilike, MO, Igbokwe, DI: Convergence theorems for asymptotically pseudocontractive maps. Bull. Korean Math. Soc. 39(3), 389-399 (2002)

10. Tang, Y, Liu, L: Note on some results for asymptotically pseudocontractive mappings and asymptotically nonexpansive mappings. Fixed Point Theory Appl. 2006, Article ID 24978 (2006)

11. Tan, KK, Xu, HK: Approximating fixed points of nonexpansive mappings by the Ishikawa iteration process. J. Math. Anal. Appl. 178, 301-308 (1993)

12. Dehghan, $\mathrm{H}$ : Approximating fixed points of asymptotically nonexpansive mappings in Banach spaces by metric projections. Appl. Math. Lett. 24, 1584-1587 (2011)

13. Osilike, MO: Iterative approximations of fixed points of asymptotically demicontractive mappings. Indian J. Pure Appl. Math. 29(12), 1291-1300 (1998)

14. Chidume, CE, Zegeye, H: Strong convergence theorems for common fixed points of uniformly L-Lipschitzian pseudocontractive semi-groups. Appl. Anal. 86(3), 353-366 (2007)

15. Shioji, N, Takahashi, W: Strong convergence theorems for asymptotically nonexpansive semi-groups in Hilbert spaces. Nonlinear Anal. 34, 87-99 (1998)

16. Suzuki, T: On strong convergence to common fixed points of nonexpansive semi-groups in Banach spaces. Proc. Am. Math. Soc. 131, 2133-2136 (2003)

17. Matsushita, S, Takahashi, W: Approximating fixed points of nonexpansive mappings in a Banach space by metric projections. Appl. Math. Comput. 196, 422-425 (2008)

18. Alber, Yl: Metric and generalized projection operators in Banach spaces: properties and applications. In: Theory and Applications of Nonlinear Operators of Accretive and Monotone Type. Lecture Notes in Pure and Applied Mathematics, pp. 15-50. Dekker, New York (1996)

19. Bruck, RE: On the convex approximation property and the asymptotic behaviour of nonlinear contractions in Banach spaces. Isr. J. Math. 38, 304-314 (1981)

20. Kruppel, M: On an inequality for nonexpansive mappings in uniformly convex Banach spaces. Rostock. Math. Kolloqu. 51, 25-32 (1997)

21. Osilike, MO, Udomene, A, Igbokwe, DI, Akuchu, BG: Demiclosedness principle and convergence theorems for k-strictly asymptotically pseudocontractive maps. J. Math. Anal. Appl. 326(2), 1334-1345 (2007)

doi:10.1186/1687-1812-2012-208

Cite this article as: Dehghan and Shahzad: Strong convergence of a CQ method for $k$-strictly asymptotically pseudocontractive mappings. Fixed Point Theory and Applications 2012 2012:208. 